\title{
ELIMINATION OF COHERENT NOISE IN A COHERENT LIGHT IMAGING SYSTEM
}

\section{GERALD J GREBOWSKY ROBERT L HERMANN HUGH B. PAULL ARNOLD R. SHULMAN}

(NASA-TA-X-70817) ELIMINATION OF COHEREAT NOISE IN A COHERENT LIGHT IHAGING SYSTEM
CSCL $20 \mathrm{~F}$ (NASA) 20 P HC $\$ 3.25$

\section{GSFC GODDARD SPACE FLIGHT CENTER}

N75-15447

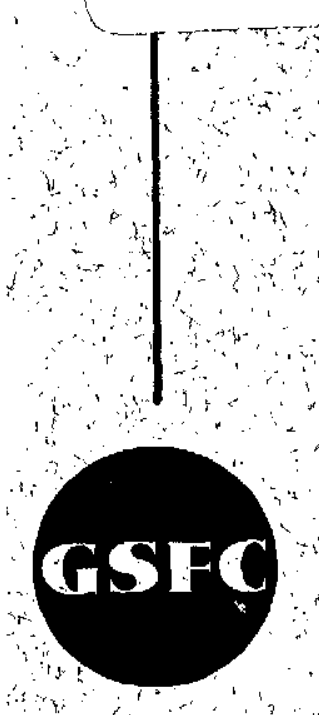

\section{MARCH 1970}


For information concerning availability of this document contact:

Technical Information Division, Code 250

Goddard Space Flight Center

Greenbelt, Maryland 20771

(Telephone 301-982-4488) 


\title{
ELIMINATION OF COHERENT NOISE
}

IN A COHERENT LIGHT IMAGING SYSTEM

\author{
Gerald J. Grebowsky \\ Robert L. Hermann \\ Hugh B. Paull \\ Arnold R. Shulman
}

March 1970

Goddard Space Flight Center

Greenbelt, Maryland 


\title{
ELIMINATION OF COHERENT NOISE \\ IN A COHERENT LIGHT IMAGING SYSTEM
}

\author{
Gerald J. Grebowsky \\ Robert L. Hermann \\ Hugh B. Paull \\ Arnold R. Shulman
}

\begin{abstract}
Optical imaging systems using coherent light introduce objectionable noise into the output image plane. Dust and bubbles on and in lenses cause most of the noise in the output image. This noise usually appears as bull's-eye diffraction patterns in the image. By rotating the lens about the optical axis these diffraction patterns can be essentially eliminated. The technique does not destroy the spatial coherence of the light and permits spatial filtering of the input plane.
\end{abstract}




\section{ELIMINATION OF COHERENT NOISE \\ IN A COHERENT IJGHT IMAGING SYSTEM}

Introduction

Dust and dirt on any lens surface will diffract light. In addition a lens usually has minute imperfections on the surfaces as well as internal to the lens. Small bubbles in the glass are not objectionable when imaging with an incoherent light source, but when imaging with a coherent light source these defects produce undesirable diffraction patterns in the output image. Using the coherent imaging system shown in Figure 1 (Refer to Appendix), a photograph was taken of the output image, Figure 2. Examination of this photograph will show many small bull's-eye patterns distributed over the image. Each of these was caused by dust, dirt on the lens surfaces, bubbles on the lenses, or imperfections on the lens surfaces. As described in this report, these undesirable noise patterns can be eliminated by rotating the lens about its optical axis.

\section{Coherent Noise Reduction by lens Rotation}

The following calculation illustrates the effect of rotating the lens on the coherent noise diffraction patterns in the image plane of the lens. Figure 3 shows the image plane of a lens. The point at which the optical axis intercepts this image plane is labeled 0. Any small dust particle on the lens will cause a bull'seye diffraction pattern in the image plane. Consider a small area dA of the noise diffraction pattern in the image plane. Let the light energy incident on $\mathrm{dA}$ be defined as $\mathrm{E}$. This energy is noise and it is desirable to have it a minimum. 
The noise energy density for this small area can be written

$$
\text { Noise energy density (No lens rotation) }=I=\frac{E}{d A} \text {, }
$$

where

$$
\mathrm{dA}=\ell \mathrm{dr}
$$

By rotating the lens about the optical axis we are able to distribute this noise energy over the area of a ring produced by rotating the area dA, i.e. instead of the noise energy striking only the area $d A$, we are able to distribute this noise energy over the larger ring area.

$$
A=\pi\left(r_{2}^{2}-r_{1}^{2}\right)=\pi\left(r_{2}+r_{1}\right)\left(r_{2}-r_{1}\right)
$$

From the geometry of the concentric circles of Figure 3 we find

$$
r_{2}-r_{1}=d r
$$

and

$$
\mathrm{r}_{2}+\mathrm{r}_{1}=2 \mathrm{r}_{1}+\mathrm{dr} \text {. }
$$

Using these relationships the expression for the area can be written

$$
\begin{aligned}
A & =\pi\left(r_{2}+r_{1}\right)\left(r_{2}-r_{1}\right) \\
& =\pi\left(2 r_{1}+d r\right) d r=2 \pi r_{1} d r+\pi d r^{2} .
\end{aligned}
$$


Since $\mathrm{dr}$ is very small, the term $\mathrm{dr}^{2}$ can be dropped, giving

$$
A=2 \pi r d r .
$$

The original energy $\mathrm{E}$ is then distributed over a ring of area

$$
\mathbf{A}=2 \pi \mathbf{r d r}
$$

The noise energy density for this ring area can be written

$$
\text { Noise energy density (with lens rotation) }=I^{\prime}=\frac{E}{2 \pi r d r} \text {. }
$$

The ratio of the energy density for the lens rotating and not rotating is

$$
I^{\prime}=\frac{\frac{E}{2 \pi r d r}}{\frac{E}{d A}}=\frac{d A}{2 \pi r d r}=\frac{\ell d r}{2 \pi r d r}=\frac{\ell}{2 \pi r} .
$$

From this we see that when

$$
\begin{gathered}
r^{\sim}, \frac{I^{\prime}}{I}=\frac{1}{2 \pi} \approx \frac{1}{6} \\
r \sim \frac{\ell}{3}, \frac{I^{\prime}}{I} \approx \frac{1}{2}
\end{gathered}
$$

If we consider $\ell$ as roughly the diameter of the noise spot in the image plane and $r$ as its distance from the optical axis, then unless the noise spot is on the optical axis (or extremely close to it), its energy will be distributed such that it can be ignored. By selection of lenses it is possible to keep the noise minimized 
by assuring that the bubbles, dust, and lens defects are not on or near the optical axis.

$\underline{\text { Results }}$

Figure 4 shows a coherent image with the usual noise diffraction patterns evident when the lens is not rotated. Figure 5 shows the image formed when the lens is rotated during the exposure. The noise diffraction patterns of Figure 4 are not evident in Figure 5.

Figures 6 through 10 demonstrate the fact that rotating the lens does not affect the spatial filtering capability of the coherent imaging system. Figure 6 shows an image of a high frequency Ronchi ruling superimposed over the transparency used for Figures 4 and 5 with the lens not rotating. Figure 7 is the image produced when the lens is rotated. Figure 8 shows the spectrum of Figure 6. Even though the lens is not rotated during the exposure, the coherent noise diffraction patterns are not evident. The reason for this is that the coherent noise diffraction patterns will appear only in the image plane and not in the spectrum plane. Figure 9 shows the Ronchi ruling of Figure 6 removed by spatial filtering (allowing only the center point of Figure 2 to be imaged). The usual coherent noise is evident in Figure 9 since the lens was not rotated. Figure 10 shows the same image as Figure 9 but with the lens rotating. The noise diffraction patterns are not evident in the spatially filtered image of Figure 10.

Fabrication of rotating lens mount

The technique used to eliminate coherent noise in coherent optical imaging systems such as shown in Figure 1 requires the lens to be rotated about the optical axis. The diffraction patterns caused by bubbles in the lens or dust on its 
surfaces can essentially be eliminated by such rotation. Extreme care must be taken to assure that the rotation of the lens is about its optical axis. This is usually quite difficult to achieve since the axis of symmetry of the lens mounting threads does not usually coincide with the optical axis. When the lens mount is rotated, any misalignment between the axis of symmetry of the lens mount and the optical axis cannot be compensated for by adjustment of the focal plane as is the case when the lens is not rotated. The adjustment to make the axis of rotation and the optical axis coincide is extremely critical.

To align the axis of rotation and the optical axis, collimated light parallel to the optical axis was focussed by the lens. As the lens is rotated the focal point should remain fixed on the optical axis. Any movement of the focal point as the lens is rotated is caused by the axis of rotation and the optical axis not coinciding. By trial and error the lens can be mounted so that the axis of rotation and the optical axis coincide.

Drawing GE 1297186, Figure 12 is the mechanical drawing for the motorized lens mount that was used. The lens which was mounted in the rotating lens mount was a Bausch and Lomb Seyser Baltar 4 "f/2 lens. Even though extreme care was taken to select and align the bearings, after assembly it was still necessary to adjust the bearing loading, endplay, and axis of rotation. These adjustments can be extremely difficult, tedious, and at times stochastic.

Summary

The noise diffraction patterns usually formed in coherent images can be eliminated by rotating the lenses used about their optical axes. This technique to eliminate the noise diffraction patterns does not destroy the coherent filtering capabilities of the coherent optical system. 


\section{APPENDIX}

Location of spectrum and image planes.

From Figure 1 it can be seen that the spectrum of the signal in the input plane appears at the image plane of the point source i.e., the spectrum location can be found from the lens formula

$$
\frac{1}{f}=\frac{1}{p}+\frac{1}{q}
$$

where

$$
\begin{aligned}
\mathbf{f}= & \text { focal length of the lens, } \\
\mathrm{p}= & \text { object distance from lens (in this case the distance } \\
& \text { of the point source from the lens), } \\
\mathrm{q}= & \text { image distance (distance from lens to image of point source). }
\end{aligned}
$$

The image plane of the signal is also found from the lens equation i.e.

$$
\frac{1}{\mathrm{f}}=\frac{1}{\mathrm{p}^{\prime}}+\frac{1}{\mathrm{q}^{\prime}},
$$

where

$$
\begin{aligned}
& f=\text { focal length of the lens, } \\
& \mathbf{p}^{\prime}=\text { distance from signal to lens, } \\
& \mathbf{q}^{\prime}=\text { distance from lens to image of signal. }
\end{aligned}
$$


This one lens coherent optical imaging system permits spatial filtering of the signal spectrum prior to imaging it in the output plane. This one lens configuration was used to check the coherent noise introduced into coherent images. 
SINGLE LENS COHERENT OPTICAL IMAGING SYSTEM

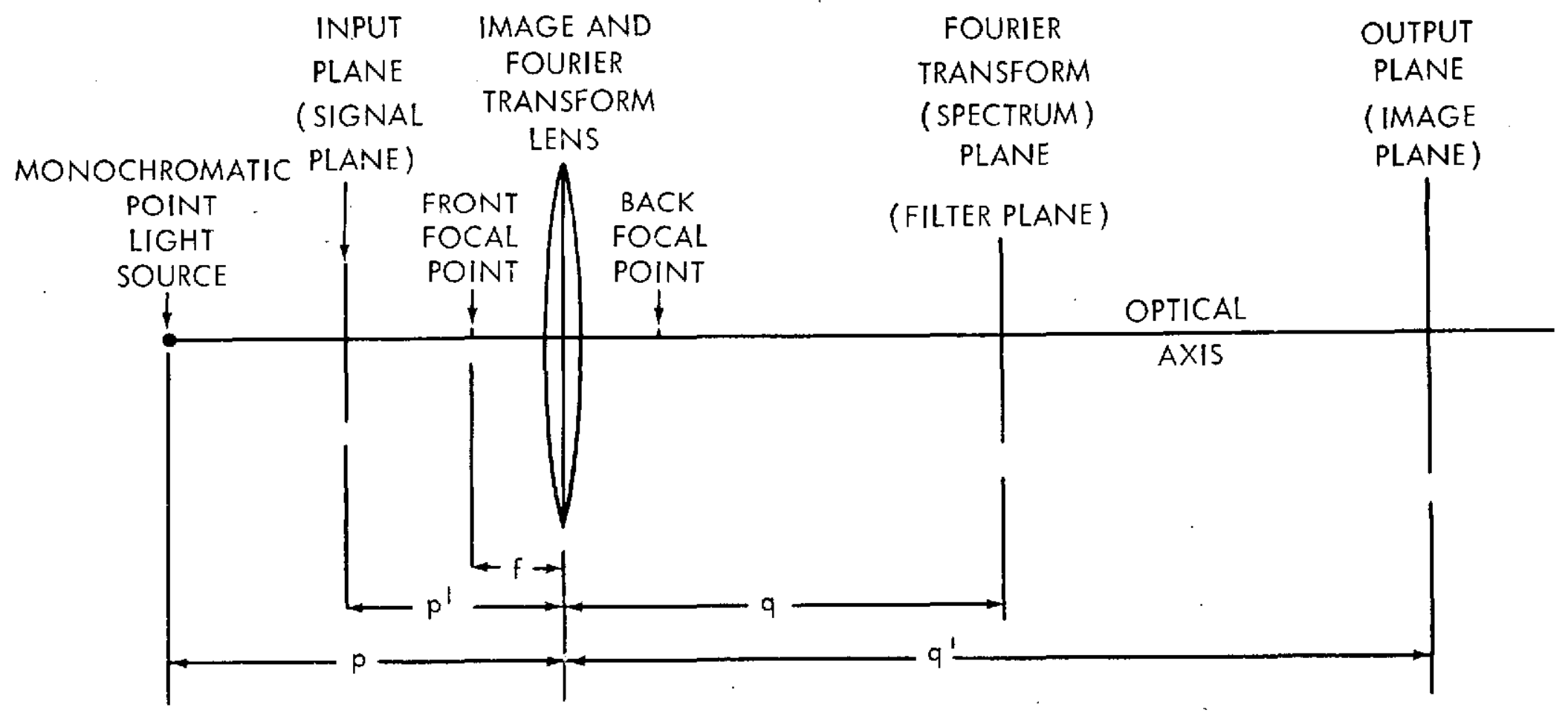

Figure 1. One lens coherent optical imaging system permitting spatial filtering of input plane 


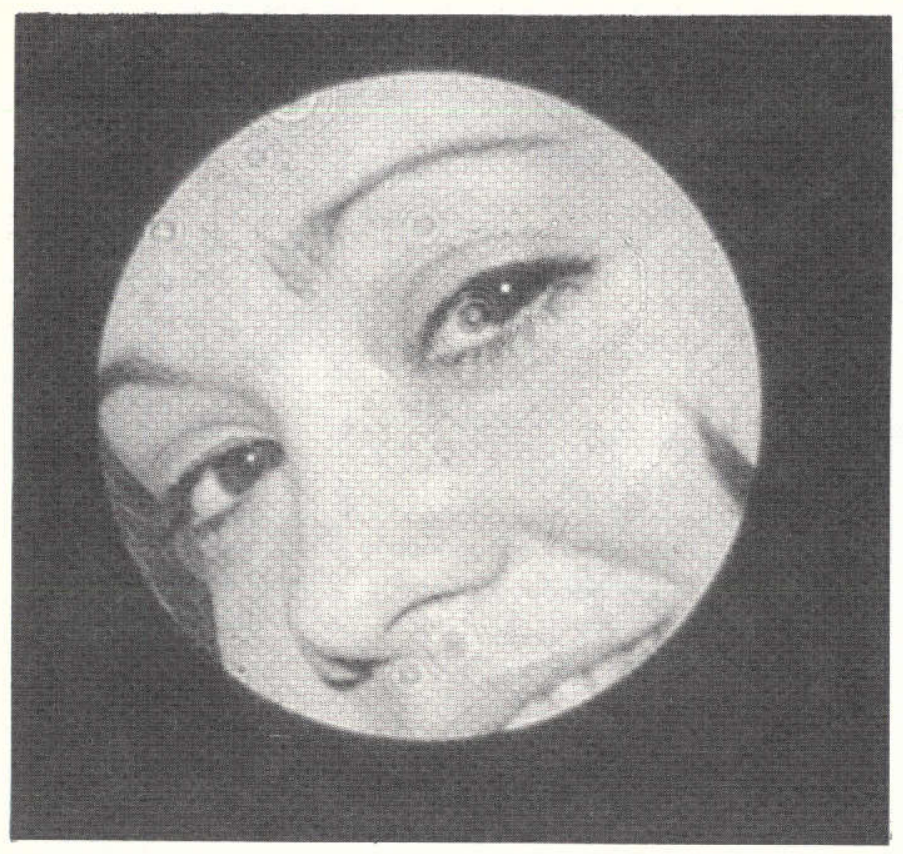

Figure 2. Coherent display usual coherent noise

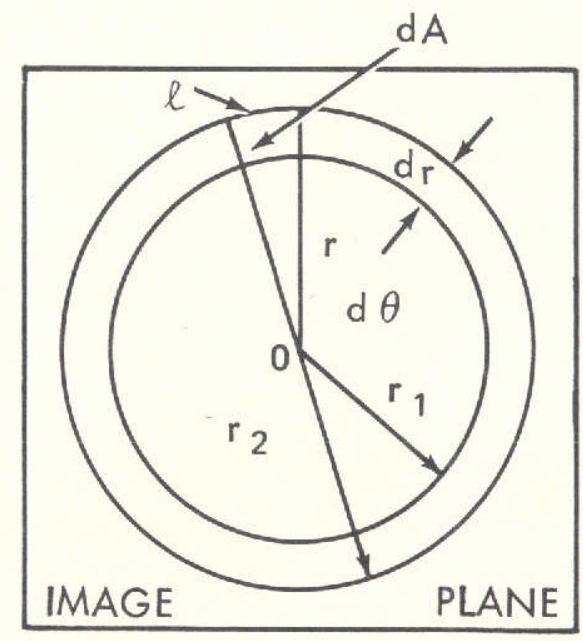

Figure 3 Rotation of noise pattern in the image plane 


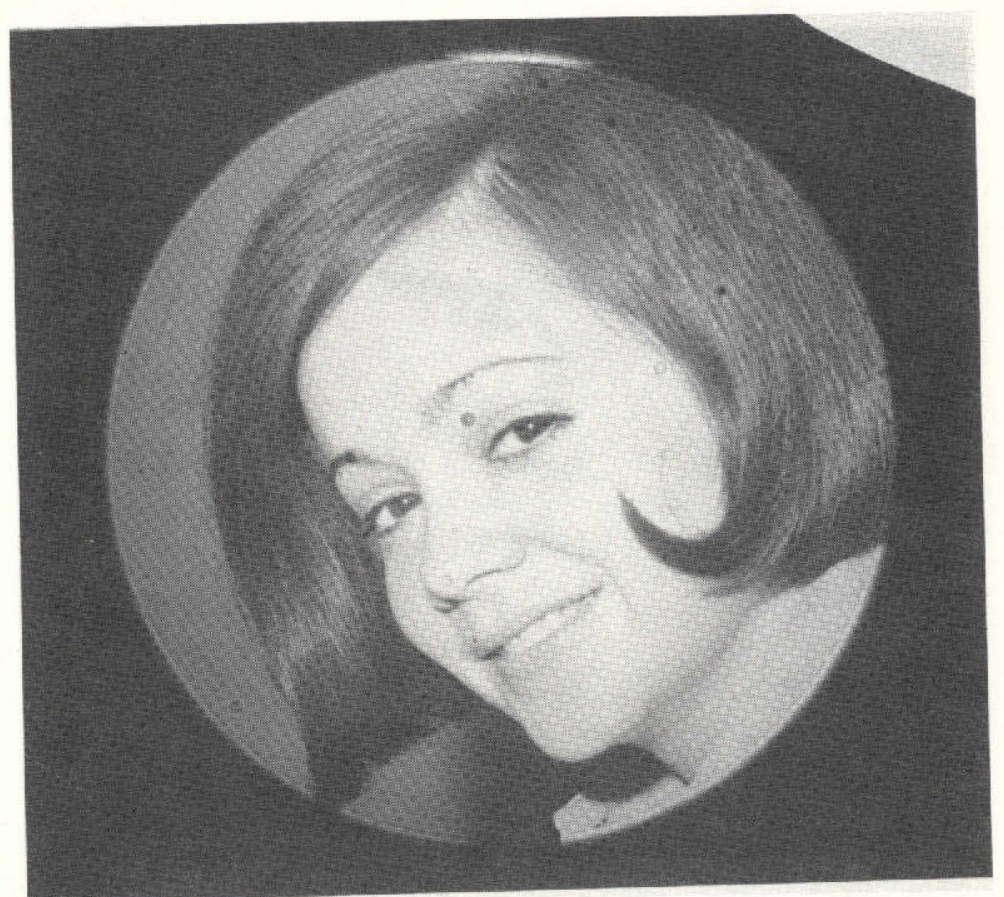

Figure 4. Coherent image formed with no lens rotation during exposure

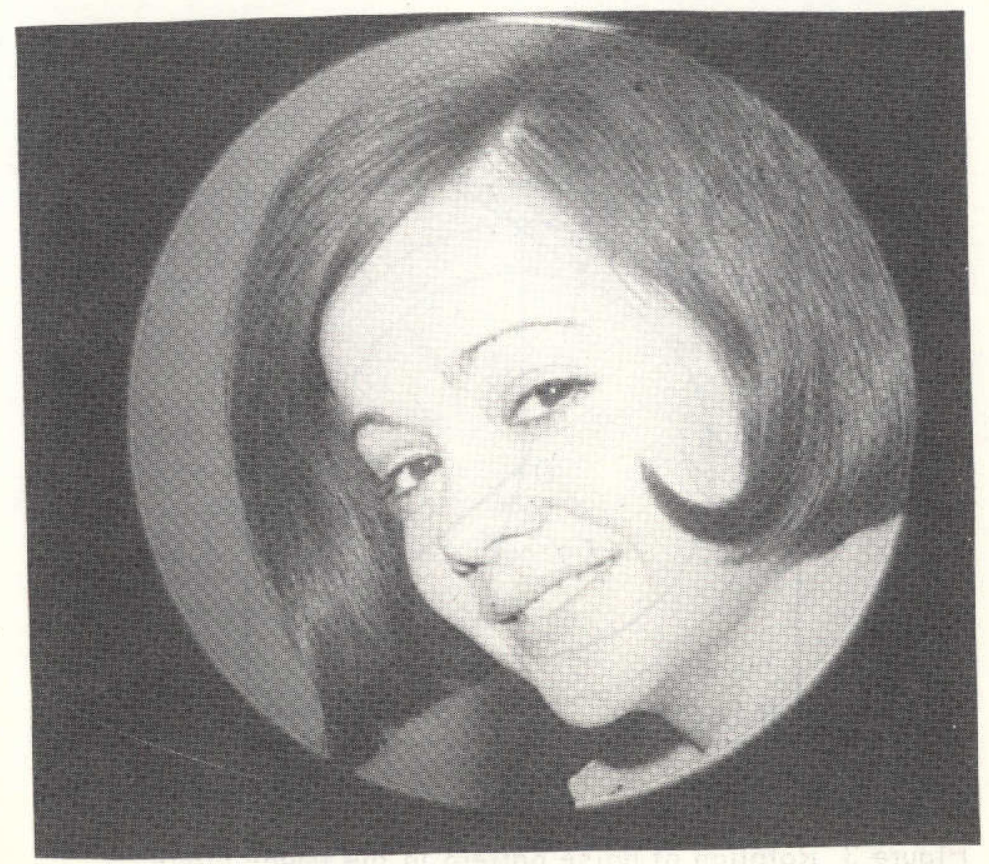

Figure 5. Coherent image with lens rotation during exposure 


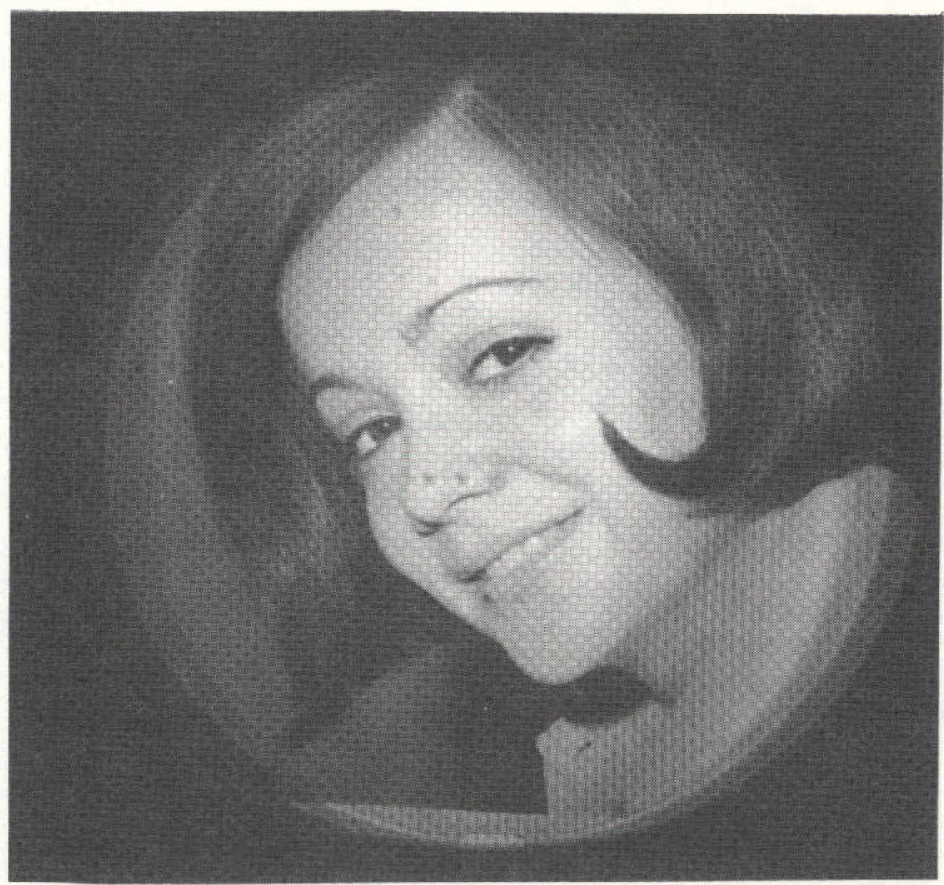

Figure 6. Coherent image of a high frequency Ronchi ruling superimposed over a transparency with no lens rotation.

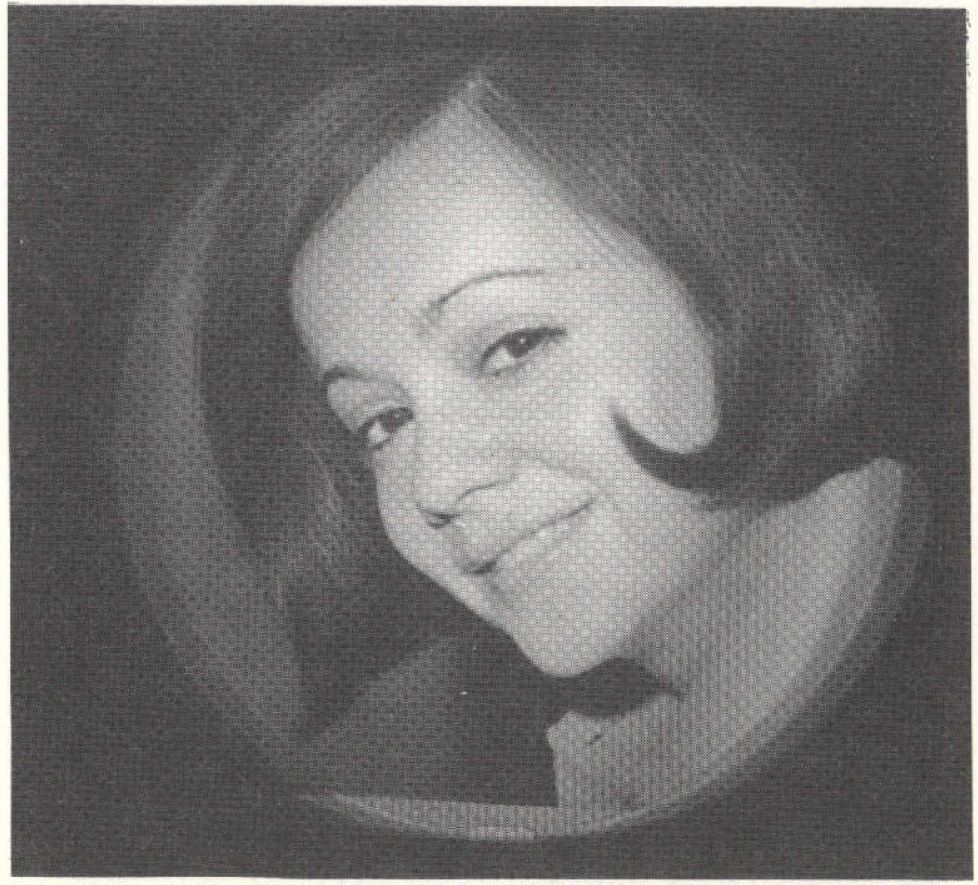

Figure 7. Coherent image corresponding to Figure 6 with lens rotation 


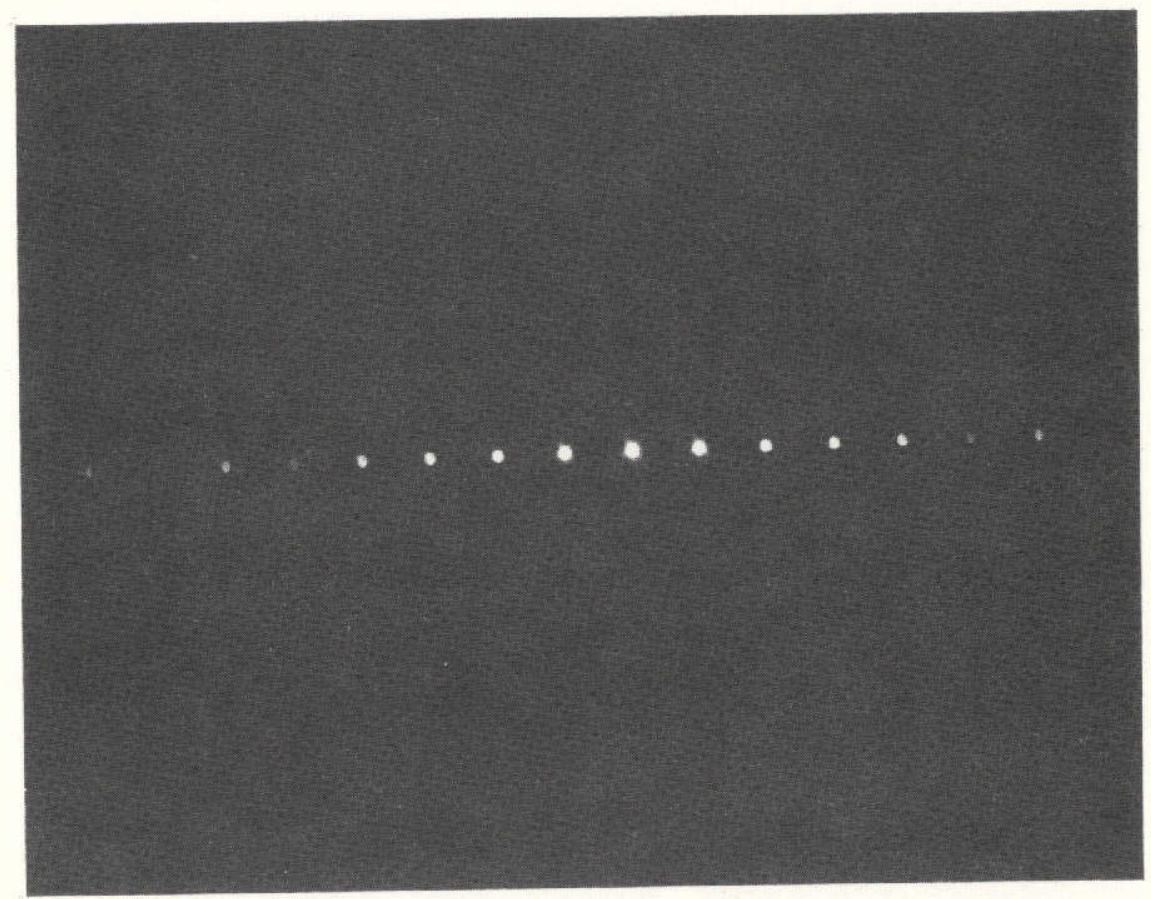

Figure 8. Spectrum of a high frequency Ronchi Ruling superimposed over a transparency (no lens rotation)

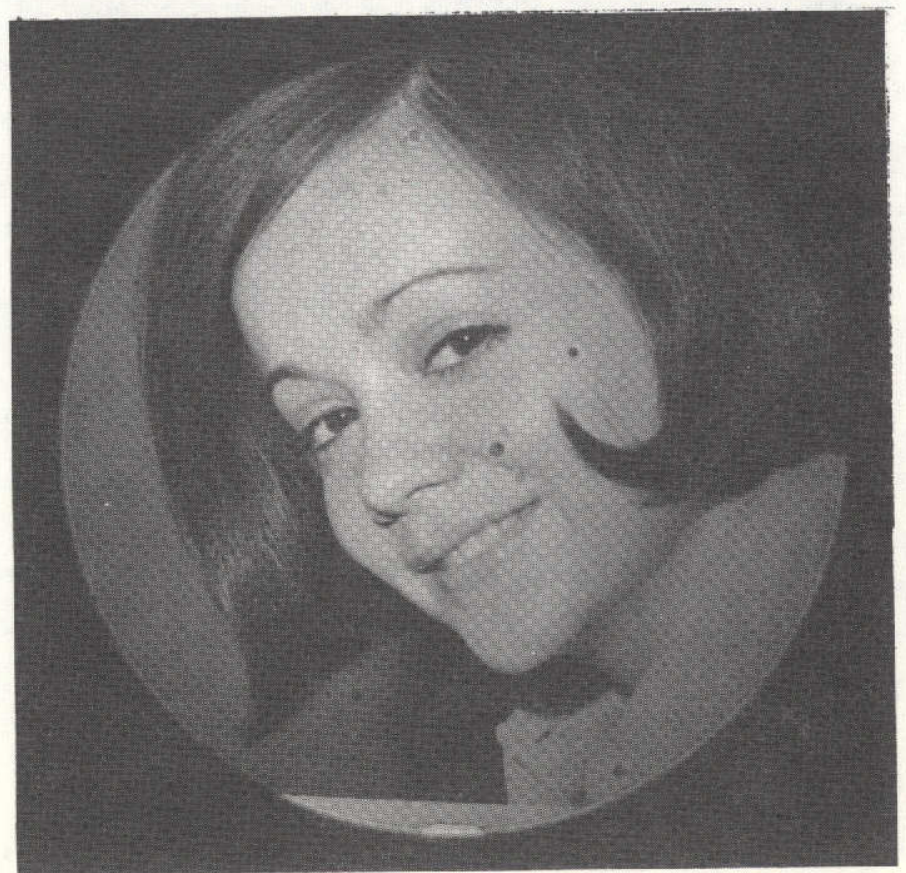

Figure 9. Spatial filtering of Fig. 6 (no lens rotation) 


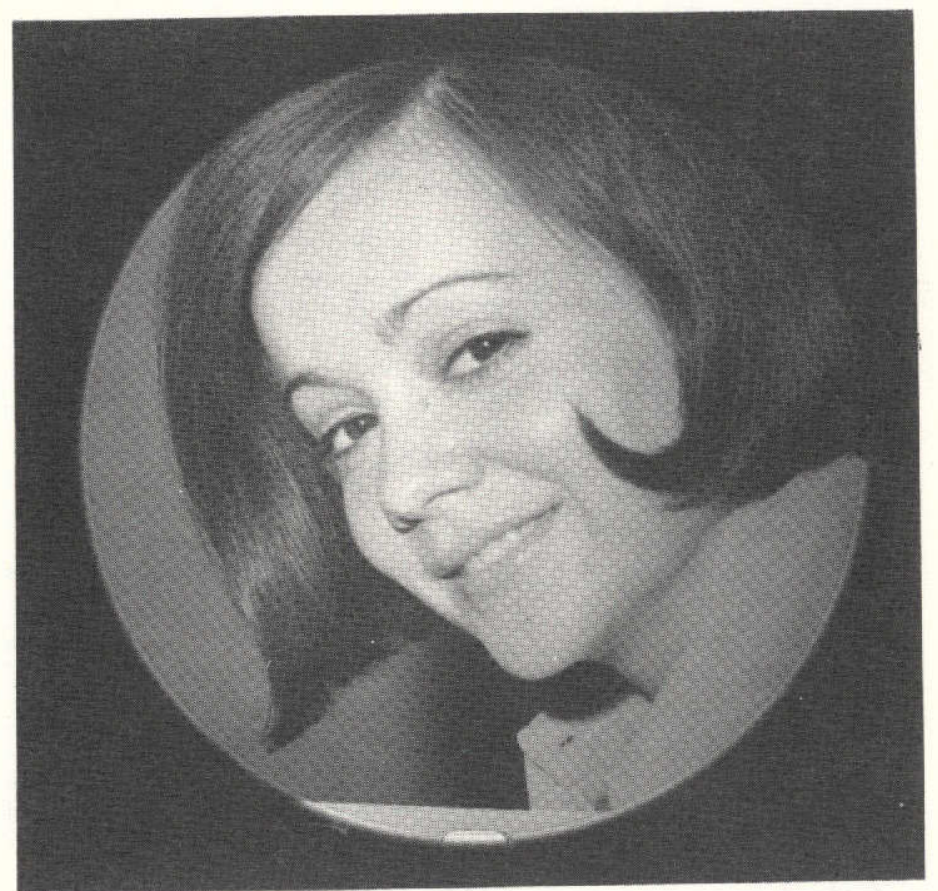

Figure 10. Spatial filtering of Fig. 6 with lens rotation

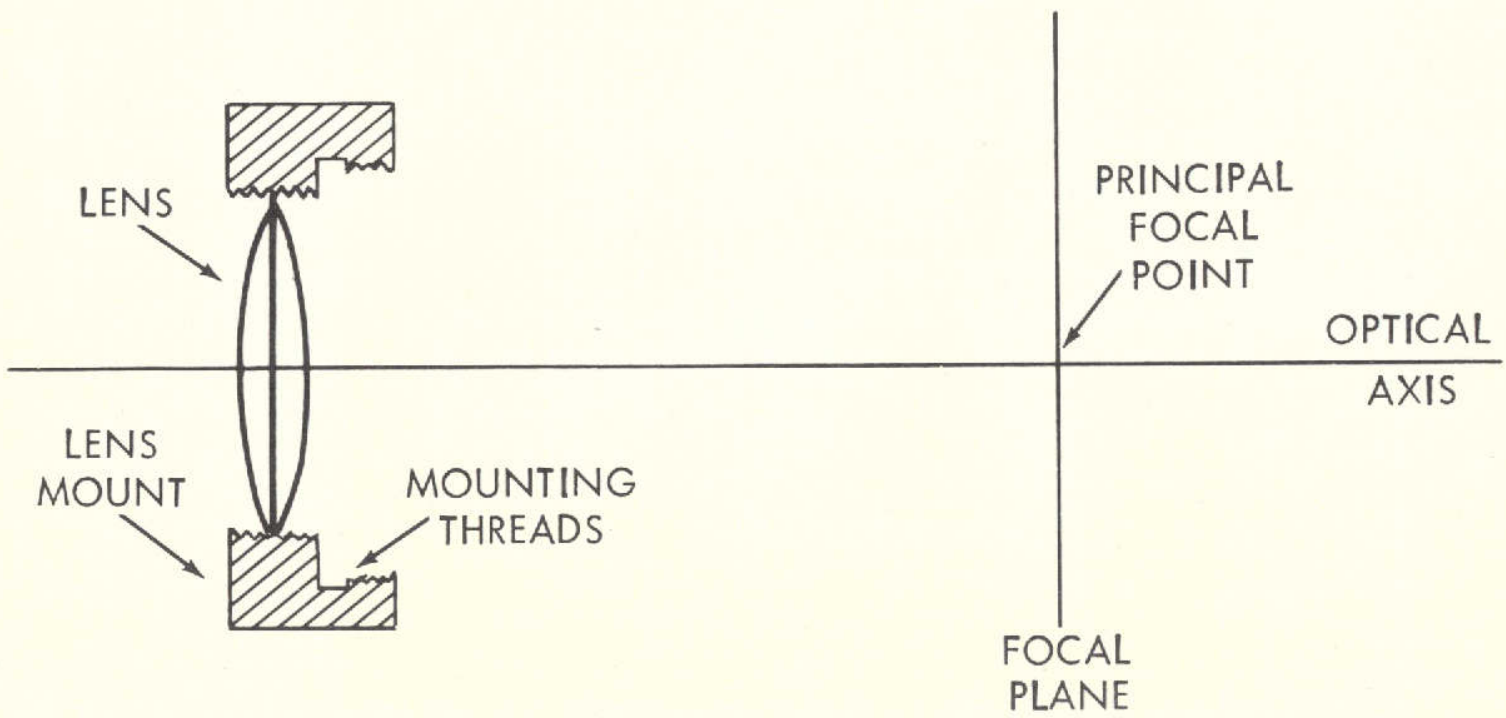

Figure 11. Lens definitions 
年

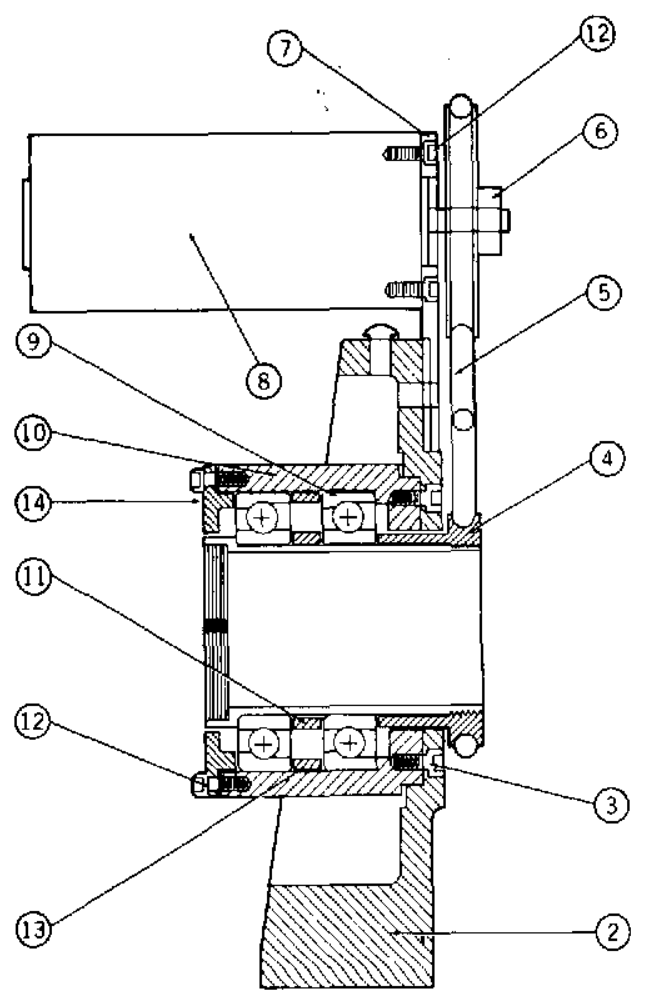

(1) ASSEMBLLY

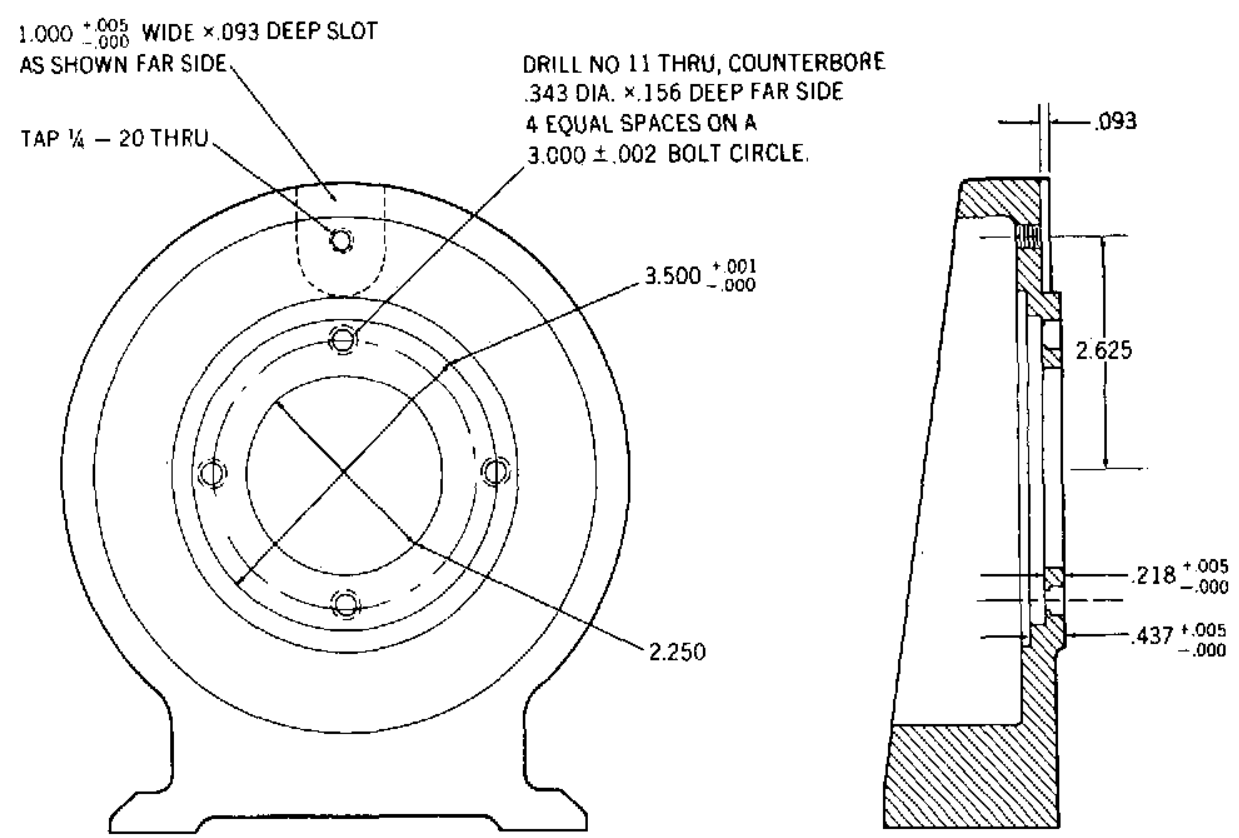

(2) MODIFY AS SHOWN
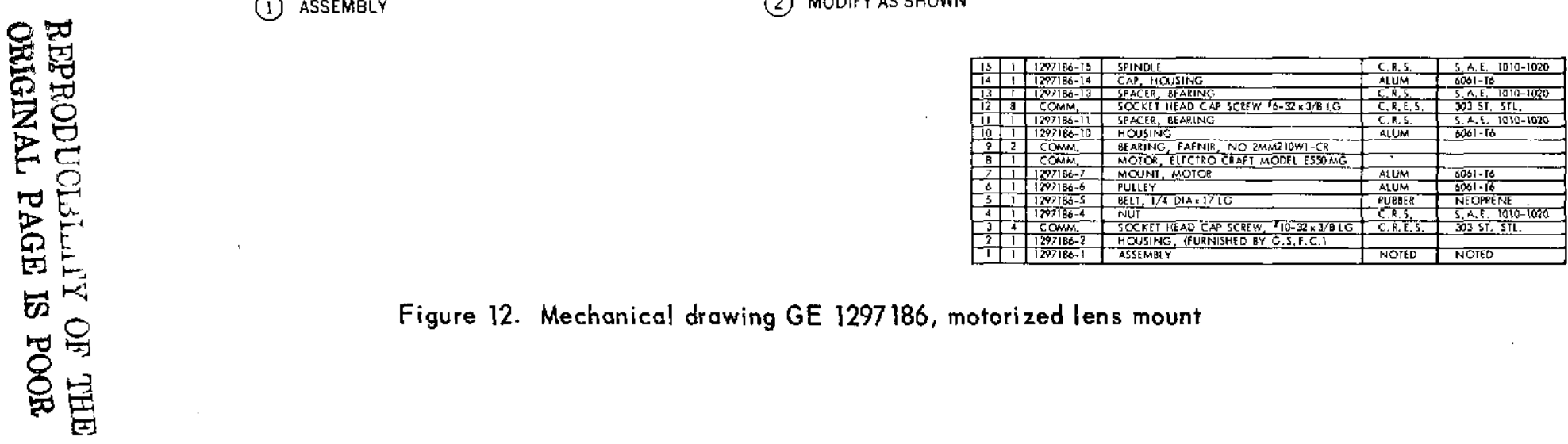

Figure 12. Mechanical drawing GE 1297186 , motorized lens mount 

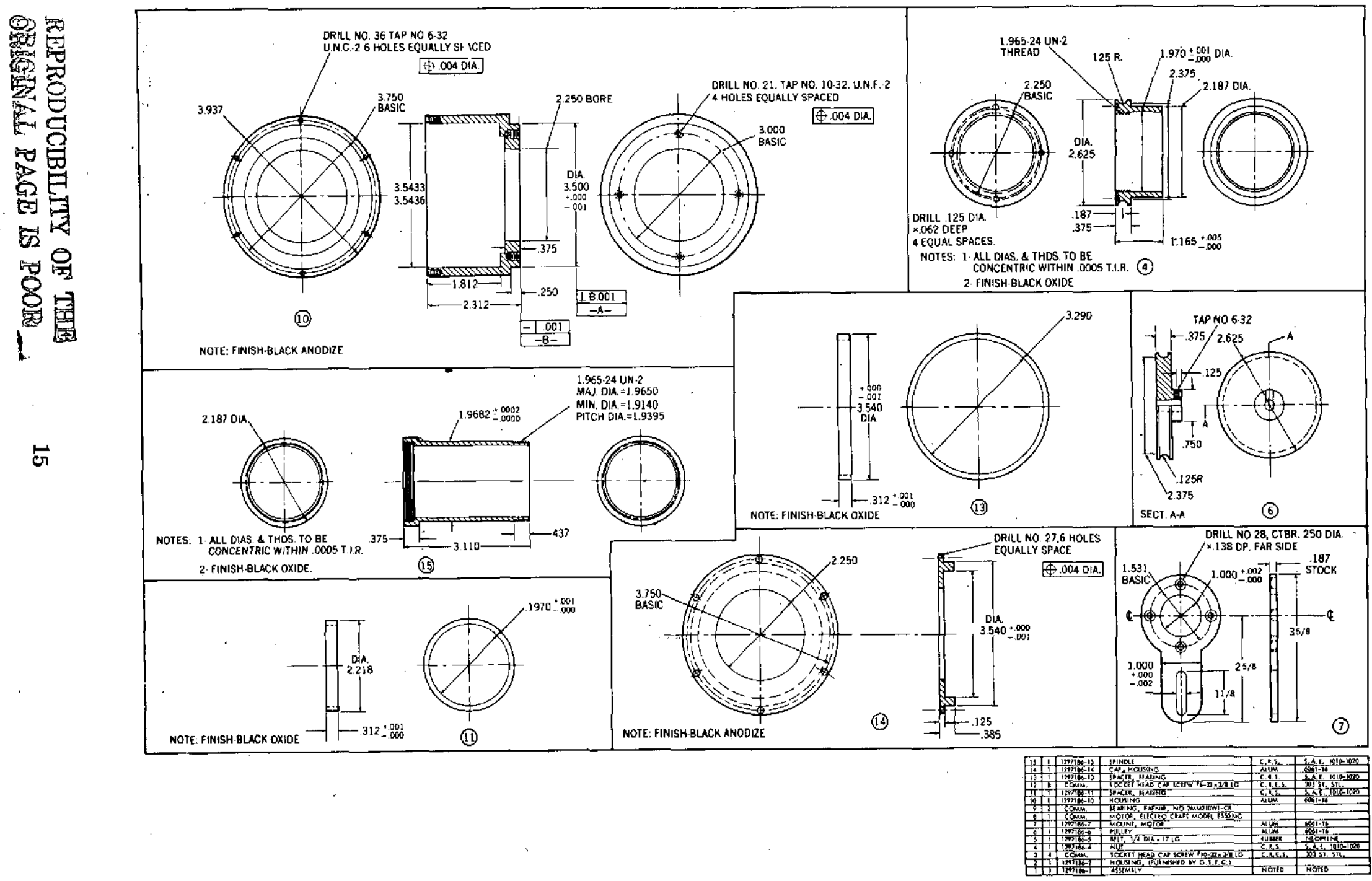

Figure 12 Continued. - Mechanical drawing GE 1297186, mortorized lens mount 


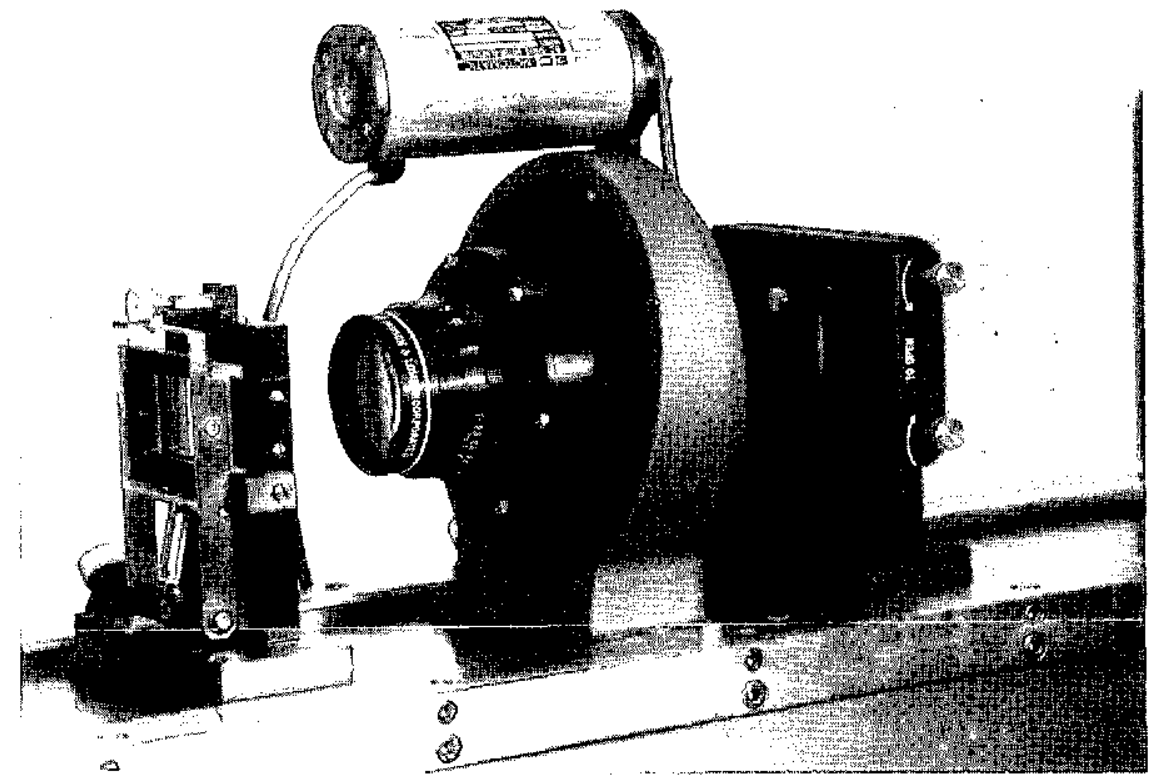

Figure 13. Oblique front view of mortorized lens mount

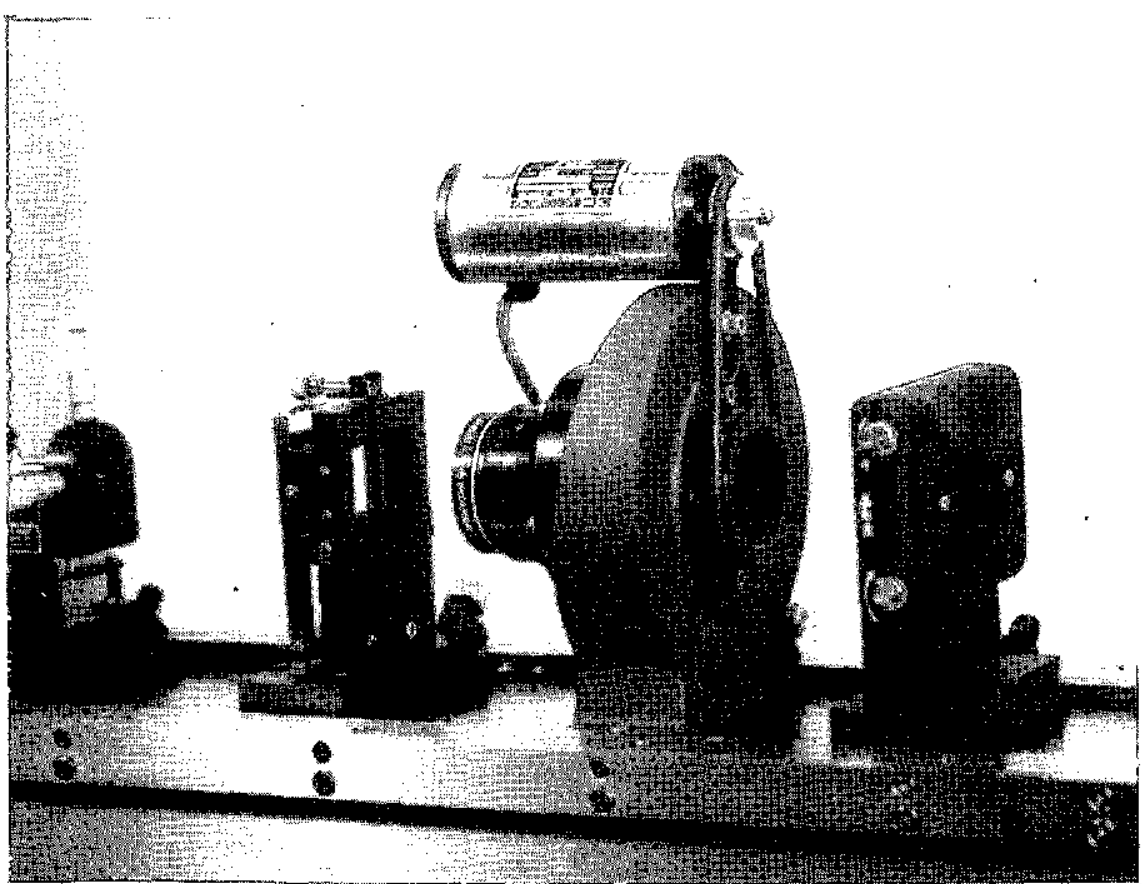

Figure 14. Oblique rear view of mortorized lens mount 